\title{
AN EXTERNAL CONTROL UNIT IMPLEMENTED FOR STIMULATOR ASIC TESTING
}

\author{
E. Noorsal ${ }^{1, *}$, K. Sooksood ${ }^{2}, H . \mathrm{Xu}^{3}$ and Z. I. Rizman ${ }^{4}$ \\ ${ }^{1}$ Faculty of Electrical Engineering, Universiti Teknologi MARA, 13500 Permatang Pauh, Pulau \\ Pinang, Malaysia \\ ${ }^{2}$ Department of Electronic, Faculty of Engineering, King Mongkut's Institute of Technology \\ Ladkrabang, 10520 Bangkok, Thailand \\ ${ }^{3}$ Department of Advanced Low Power Solution, Texas Instrument Deutschland, Freising, \\ Germany \\ ${ }^{4}$ Faculty of Electrical Engineering, Universiti Teknologi MARA, Dungun, Terengganu, Malaysia \\ Published online: 10 November 2017
}

\begin{abstract}
This paper presents the design and development of an external control unit (ECU) for a stimulator ASIC testing purposes. The ECU consists of a graphical user interface (GUI) from the PC, a data transceiver and a power transmitter. The GUI was developed using MATLAB for stimulation data setup. The data transceiver was designed using hardware description language (HDL) Verilog code and was implemented in a Virtex-II Pro FPGA board. The overall stimulator ASIC design architecture and its operation for an epiretinal implant application are briefly explained to correlate with the ECU's design requirements. The flexible multichannel stimulator ASIC was successfully fabricated in a $0.35 \mu \mathrm{m}$ AMS HVCMOS technology. Conducted simulation and measurement results on stimulation waveform generation, supply voltage compliance and external control of supply voltage adaptation validate the functionality of the designed ECU and the stimulator ASIC.
\end{abstract}

Author Correspondence, e-mail: emilia.noorsal@ppinang.uitm.edu.my doi: http://dx.doi.org/10.4314/jfas.v9i6s.54 
Keywords: external control unit; data transceiver; stimulator ASIC; retinal prosthesis; epiretinal implant; stimulation waveform; Manchester data; voltage compliance.

\section{INTRODUCTION}

Blindness has a severe impact on an individual's lifestyle and on socioeconomics. A person who is afflicted with total or partial blindness is often unable to independently perform rudimentary tasks. A visual prosthesis is a device that is used to provide blind patients with a sense of vision by electrically stimulating the neural cells in the visual pathway. To date, much research has been pursued on the retinal prosthesis. In recent years, two main research areas on retinal prostheses have been pursued, namely the epiretinal implant [1-7] and the subretinal implant [8-10]. The primary goal of these prostheses is to bypass the damaged photoreceptors and to electrically stimulate the remaining cells of the retina for visual excitation. Fig. 1 illustrates a system overview of an epiretinal implant with its components [1]. The external system mainly consists of a camera and a digital image processor. The captured image from the camera is digitally processed and converted into encoded stimulation data by the processor before it is transmitted wirelessly to the implant.

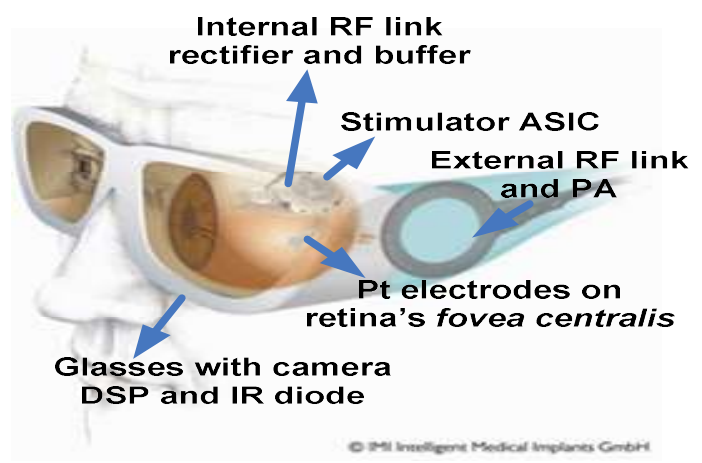

Fig.1. System overview of an epiretinal implant [11]

This implanted system, which is telemetrically powered, consists of the stimulator electronics and the stimulation electrodes. The stimulator electronics consists of a built-in stimulator ASIC together with several passive components for the power management. An external control unit (ECU) is typically employed in an implantable micro-stimulator system to program the intended stimulus parameters and to monitor the status of the implant chip for complete safety closure [12-15]. The examples of important safety features that requires ECU's surveillance are the internal and compliance of the supply voltage, the electrode impedance, the non-accomplished charge balancing, the unwanted-fault current into the electrode due to shorted switch, the 
decrement in supply voltage due to misalignment of the RF link and the corrupted received data $[1,12,14-16]$. The desirable features of an ECU with multichannel stimulator is high data rates for stimulation programming, power delivered through RF inductive link, monitoring and surveillance for complete safety closure and portability [14, 17]. Therefore, the implementation of a reliable ECU is indispensable for the implantable micro-stimulator system.

In this work, the design and implementation of the ECU for an epiretinal stimulator ASIC are discussed and illustrated. The ECU was mainly developed to test the functionality of the fabricated stimulator ASIC as well as for monitoring purpose. This paper is organized as follows. Section 2 provides an overview of the overall system architecture for multichannel stimulator ASIC, the implant chip monitoring, the serial backchannel data transfer operations and the voltage adaptation protocol. Then, section 3 elucidates the design overview of the ECU, the internal architecture of a data transceiver in the ECU and the data transceiver's protocol from the ECU to the stimulator ASIC. Section 4 discusses the simulation results of the data transceiver module and the measurement results of the functionality and bidirectional communication link between the ECU and the fabricated stimulator ASIC. Lastly, section 5 presents the conclusion.

\section{METHODOLOGY}

\subsection{Overview of Stimulator ASIC Design Architecture}

The proposed stimulator ASIC design architecture for a 1024 channel epiretinal implant is depicted in Fig. 2. The stimulator ASIC was designed using hardware description language (HDL) Verilog code and was implemented in a $0.35 \mu \mathrm{m}$ AMS HVCMOS technology. The internal design architecture of the stimulator ASIC mainly consists of Global and Local Function modules. The global function module consists of a reconfigurable HV power management unit, an optical receiver, a clock recovery, an ADC, a load modulator and a Global Stimulation Control Unit (GSU). The local function module consists of an extendable number of distributed Local Stimulation Units (LSUs). The implant ASIC is powered by a $13.56 \mathrm{MHz}$ RF inductive link [1, 11, 18-19]. The HV power management unit, rectifies the RF input by employing several integrated rectifiers in the circuit [18]. From the power regulator unit, two main supply voltages are provided namely a $3.3 \mathrm{~V}$ supply for all low-voltage operations $\left(\mathrm{V}_{\mathrm{DD}}\right)$ and an adjustable high-voltage supply $\left(\mathrm{V}_{\mathrm{DDP}}\right)$ of up to $20 \mathrm{~V}$, which is used for the stimulator outputs. The ECU program the intended stimulus parameters by sending the stimulation data (encoded Manchester) 
to the stimulator ASIC at a data rate of approximately $\sim 1 \mathrm{Mb} / \mathrm{s}$ via an optical link. Details of the stimulation and data packet protocols can be found in [11].

The clock recovery module, converts the received RF signal into a $13.56 \mathrm{MHz}$ clock, which is used by the GSU as a master clock. The function of the GSU is to receive the serial encoded Manchesterdata from the Optical Receiver and to control the implant operation. The output signals from the GSU module, which include stimulation commands ("stim_cmd"), a slow system clock ("clk_gbl”) at $1 \mathrm{MHz}$, the address bus and the local stimulation data are connected globally to all LSUs. Each LSU comprises of a digital pixel control unit (PCU), a 5-bit current steering DAC and a 1:4 demultiplexed output HV current driver, which includes a voltage-compliance monitor, charge balancers, a sample and hold $(\mathrm{S} \& \mathrm{H})$ circuit for electrode impedance measurements. After the reception of stimulation data, the GSU will program the local stimulation data to the addressed LSUs. The LSU then provides a stimulation pulse current to the attached electrodes. The 256 number of LSUs are arranged in an array and addressed using row and column address decoders.

For electrode charge balancing safety, the global charge balancing circuit (global balance) updates the status of the electrode balancing from the activated LSUs to the GSU. This is to ensure that the implant ASIC is safe from any unbalance accumulated charge, which exist in the electrode after each stimulation [20]. Additionally, global compliance monitoring from all LSUs is conducted by the Global Compl module, which receives feedback compliance signals from all LSUs. The voltage compliance signal from each LSU ("compl") indicates the status of the supply voltage compliance for each LSU. 


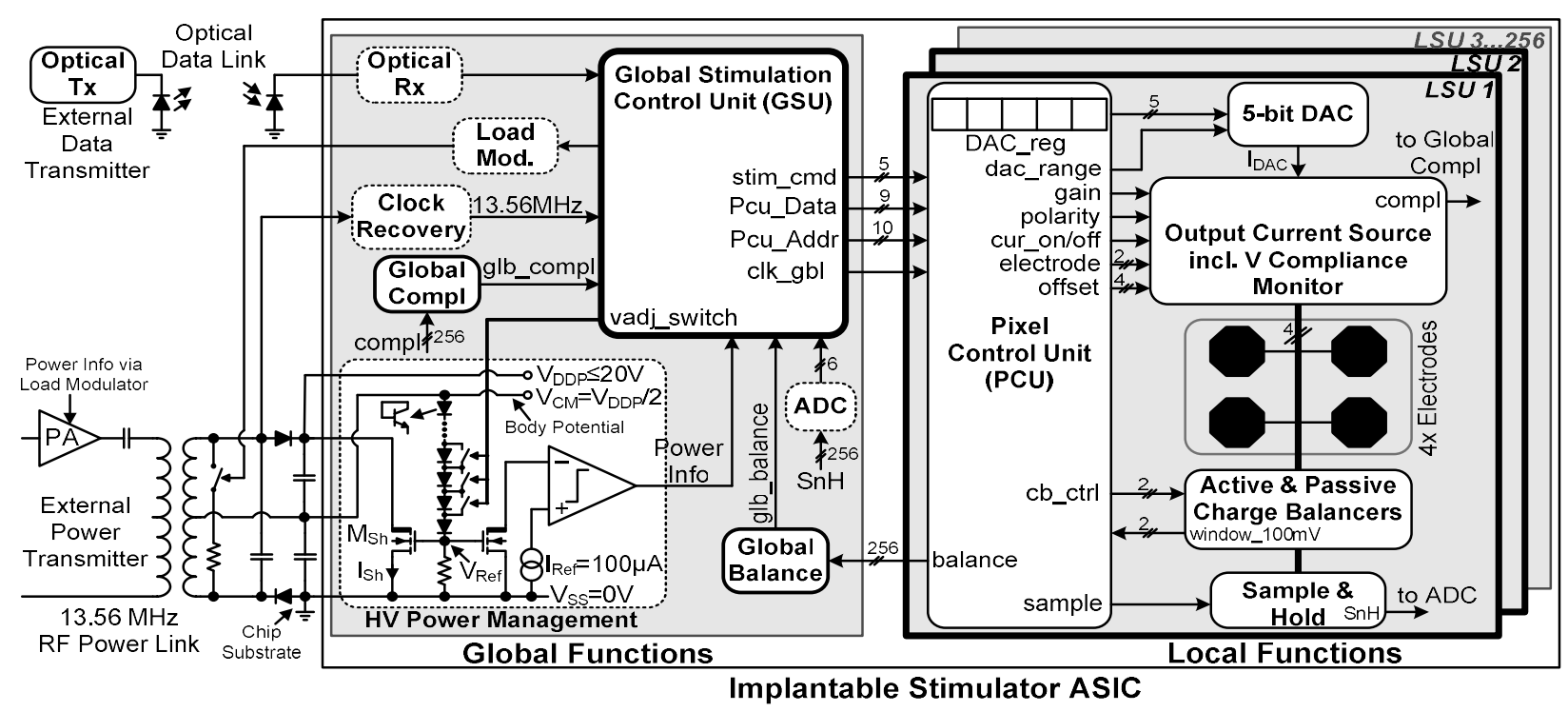

Fig.2. The proposed overall epiretinal stimulator system architecture, which consists of global and locally distributed functions [11]

The status or conditions of epiretinal implant are back communicated to the ECU through the Load Modulator and RF inductive link for monitoring purposes. The examples of epiretinal implant conditions that are back communicated to the ECU are the global voltage compliance problem, the global non-accomplished charge balancing, the electrode impedance, the internal power condition of the shunt regulator and the data packet error. These epiretinal implant conditions are first converted into 16-bit backchannel data are then processed by the GSU to produce pulse position coded signal. The pulse code signal triggers the switch through the Load Modulator for RF inductive link transmission. Details backchannel data processing and serial pulse code generation by the GSU can be referred here [12].

In this design work, the supply voltage $\left(\mathrm{V}_{\mathrm{DDP}}\right)$ can be adjusted and adapted externally by the ECU according to the currently running of stimulation compliance voltage. The ECU will send the voltage adaptation data via optical link (either voltage increase or voltage decrease) to the stimulator ASIC depending on the feedback information of the back-communicated data. Once the GSU receives the voltage adaptation data ("vadj_switch") from the ECU, it will selectively short out the reference diode string in the HV Power Management unit for shunt current adjustment. The 4-bit of "vadj_switch" register, which is directly connected to the diode strings from the GSU, controls the increment and decrement of the $\mathrm{V}_{\text {DDP }}$. To decrease the high supply voltage, the diode switches are shorted out by shifting binary "1" into the 4-bit "vadj_switch", 
starting from LSB towards the MSB (from minimum "4b'0001" to maximum " $4 \mathrm{~b} 1111$ " decrement). In contrast, to increase the supply voltage, the diode switches are opened by shifting binary "0" into the 4-bit "vadj_switch", starting from MSB towards the LSB (from minimum “4b'0111" to maximum " $4 \mathrm{~b} 0000$ " increment).

After power on reset, the ECU starts to monitor the compliance supply voltage of the implant. For example, if no compliance problem is detected for a stimulation time frame of $100 \mathrm{~ms}$, the ECU will send a voltage decrease data to decrease the supply voltage because it is assumed that the $\mathrm{V}_{\mathrm{DDP}}$ is too high for the currently running stimulation. Thereafter, the GSU decreases the shunt reference by shorting out one diode. Thus, $\mathrm{I}_{\mathrm{Sh}}$ is increased and the $\mathrm{V}_{\mathrm{DDP}}$ is reduced accordingly. The process of decreasing the supply voltage at every $100 \mathrm{~ms}$ is repeated until the implant reports a voltage compliance problem to the ECU. For this, the compliance monitor in every output stage detects and reports any output voltage compliance problem ("compl"). It should be noted that the monitoring time frame of $100 \mathrm{~ms}$ is not fixed. It is selected based on the stimulation time frame, in which the external monitoring time frame should not be less than one stimulation time period and should not be longer than the frequency of the external camera movement $(1-10 \mathrm{~Hz})$ in the epiretinal implant system. This is because the camera movement has a direct effect on the changes of the ambient light intensity that enters the camera.

After reception of the backchannel data and the detection of a voltage compliance problem in the epiretinal implant by the ECU, a voltage adjust data to increase the supply voltage is sent from the ECU to the epiretinal implant. The GSU will then increase the supply voltage accordingly by opening a diode switch in the shunt regulator. Thereafter, the ECU assumes that the implant has found an optimal compliance voltage and stops sending the voltage decrease data at every $100 \mathrm{~ms}$. The next voltage decrement monitoring is carried out every 1s. If subsequently any of the local voltage-compliance monitors detect a too small $\mathrm{V}_{\mathrm{DDP}}$, the GSU immediately reports to the ECU and a new voltage increase data is sent to readjust the shunt reference by opening one diode of the string to increase the $\mathrm{V}_{\mathrm{DDP}}$ accordingly. Thus, the supply voltage for the output current driver is always controlled to adjust the supply voltage just high enough for all currently running stimulations over time. Thus, the power efficiency of the stimulator ASIC is increased.

\subsection{Design Methodology and Implementation of an External Control Unit}

Fig. 3 depicts the ECU test measurement setup for the stimulator ASIC. The ECU mainly consists of a graphical user interface (GUI) from the PC, a data transceiver using a Virtex-II Pro FPGA 
board and a power transmitter. The main functions of the ECU are to provide stimulation data to the stimulator ASIC for stimulation operation, to monitor the status of the stimulator ASIC and to provide external closed-loop power management for complete safety closure. The test measurement of the fabricated stimulator ASIC is started by setting the intended stimulation data in GUI. The intended stimulation data are then transferred to the FPGA board for temporary data storage through the USB connector. Once completed, the stored stimulation data are then retransmitted to the stimulator ASIC at a data rate of $\sim 1 \mathrm{Mbps}$ using the Manchester encoded data. It is to note that $\sim 1 \mathrm{Mbps}$ data rate is chosen to emulate the actual data transfer using the optical link in the epiretinal implant application [1]. The stimulation data can be sent one at a time or at a stimulation frame rate of 8 different frequencies ranging over $30 \mathrm{~Hz}, 60 \mathrm{~Hz}, 100 \mathrm{~Hz}, 200 \mathrm{~Hz}, 300 \mathrm{~Hz}$, $500 \mathrm{~Hz}, 1 \mathrm{kHz}$ and $2 \mathrm{kHz}$ for repetitive stimulations. Fig. 4 depicts an example of the actual test measurement setup of the ECU with the stimulator ASIC.

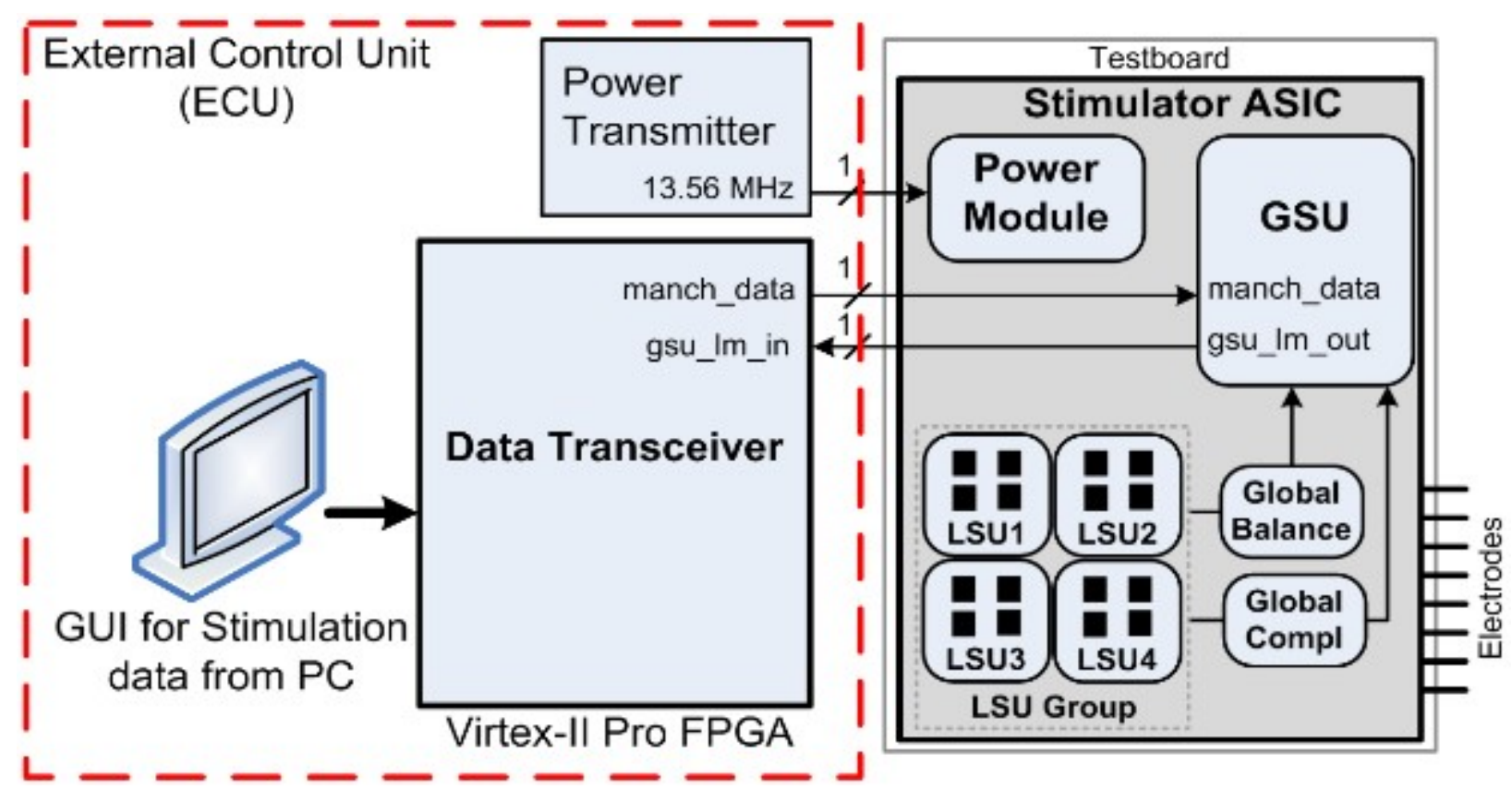

Fig.3. ECU test measurement setup for stimulator ASIC 


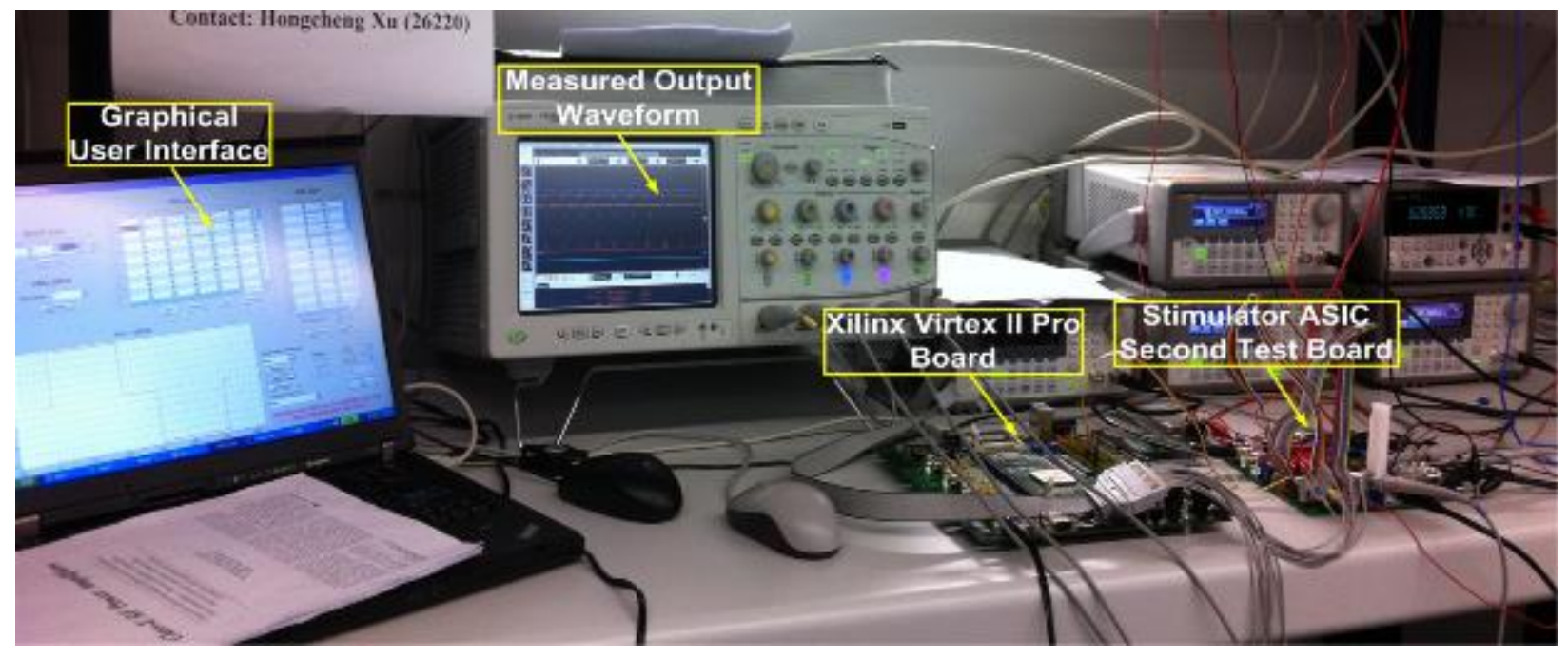

Fig.4.Actual ECU test measurement setup for stimulator ASIC

\subsection{Stimulation Data Setup and Processing}

The stimulation data are first prepared according to the intended stimulation pattern that is set by the users. A graphical user interface (GUI) [22-23] has been developed using MATLAB software to assist the setup of the intended stimulation data such as local amplitude profiles and global timing settings as depicted in Fig. 5. The stimulation data are first mapped into binary representation. Thereafter, the stimulation data are harvested into several data packets with appended 16-bit CRC signatures. The intended stimulation profile can also be observed in this GUI by pressing the plot button. After the user is satisfied with the stimulation wave pattern settings, the stimulation data are sent to the FPGA according to the data protocol rules. For PC to FPGA board communication, the complete data packet is then divided into an 8-bit of unsigned integer value and it is appended with the last byte, which is set to the value 255 to indicate the end of the data packet. The 8-bit data are then sent to the FPGA through the USB connection. 


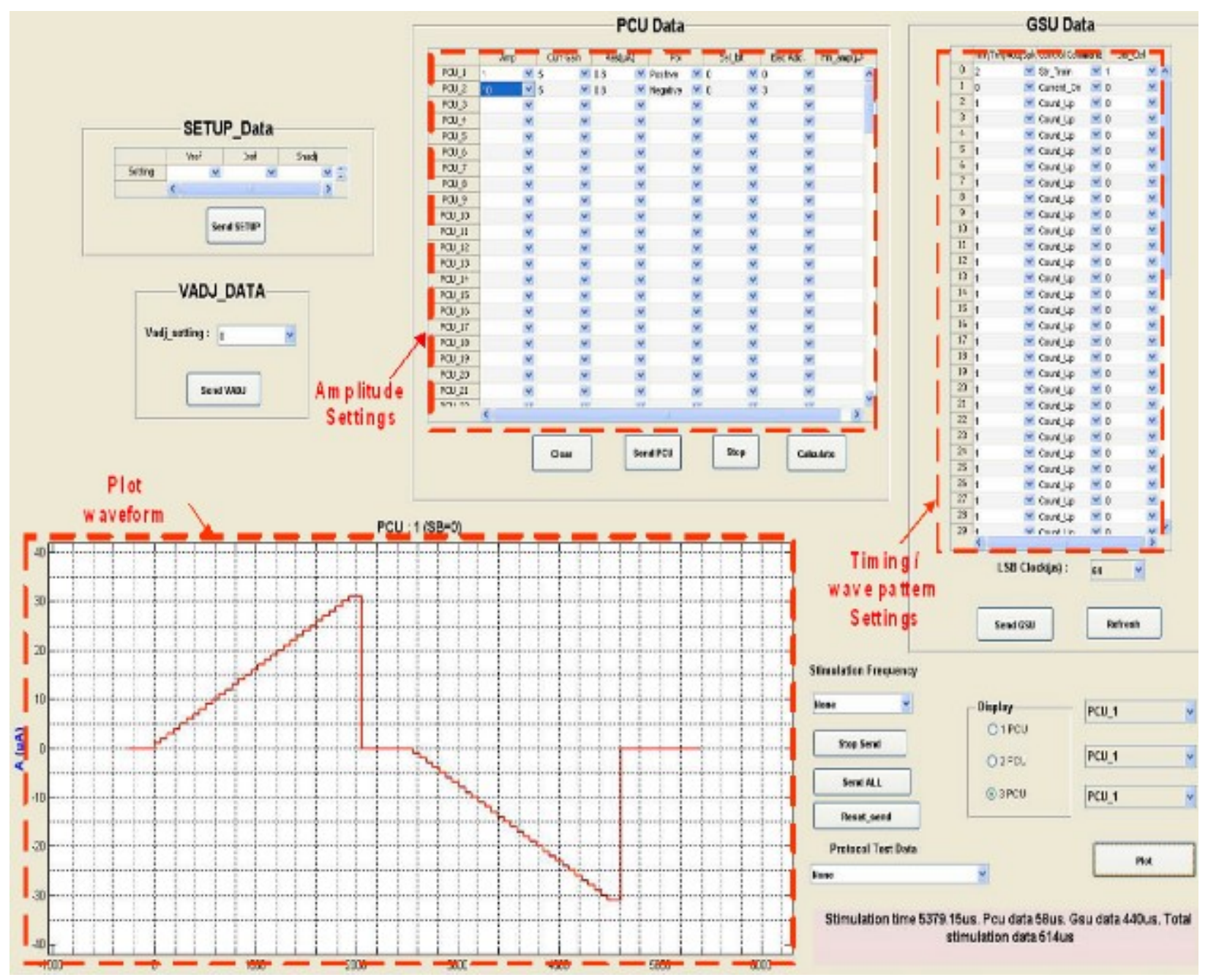

Fig.5. Graphical user interface for stimulation data setup

\subsection{Data Transceiver Design Architecture}

The data transceiver was designed using the HDL Verilog code and was implemented in Virtex-II Pro FPGA board. The main functions of the data transceiver are to temporarily stored the received data from the $\mathrm{PC}$, to provide serial Manchester data at $\sim 1 \mathrm{Mbps}$ to the stimulator ASIC, to decode the backchannel data from the stimulator ASIC and to provide a closed-loop external control mechanism for stimulator ASIC supply voltage adaptation. For the stimulator ASIC test measurement setup, the output signals from the Data Transceiver are connected to the stimulator ASIC. 


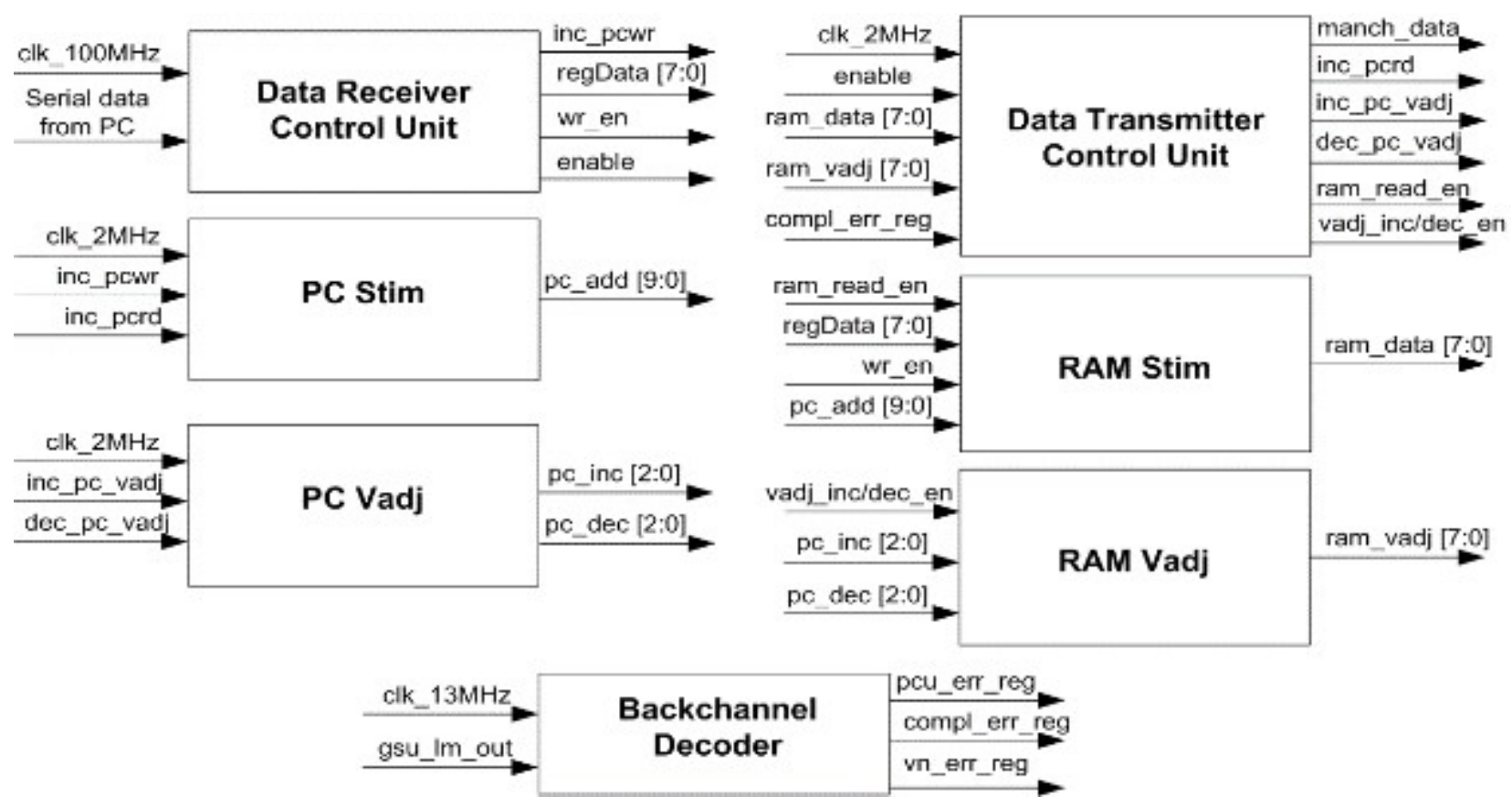

Fig.6. Data transceiver implemented in the FPGA

The internal architecture of the data transceiver as depicted in Fig. 6 consists of several sub-modules, which include a data receiver control unit, a PC Stim, a data transmitter control unit, a RAM Stim, a RAM Vadj and a backchannel decoder. The data receiver control unit controls the reception of serial data from the PC into the FPGA board. Therefore, the data receiver control unit provides the 8-bit received data ("regData") from the PC, a 1-bit "wr_en" signal to enable the writing process of the 8-bit "regData" into the RAM Stim and a 1-bit "inc pcwr" signal to increase the program counter address (PCStim). The PC Stim address is reset to its starting location when the received 8-bit "regData" has the value of 255, which indicates the end of the data packet. The PC Stim acts as an address pointer during the writing and reading of the 8-bit data into and from the RAM Stim. The RAM Stim is a register file with an organization of 8-bit $\times$ 1024 rows. Writing of the 8-bit "regData" into the RAM Stim uses a high clock frequency ("clk_100MHz") while reading of the 8-bit data uses a low clock frequency ("clk_2MHz"), which is the clock frequency for the Manchester data transmission. In contrast, the PC_Vadj, acts as an address pointer during the reading of the 8-bit data from the RAM_Vadj. The RAM_Vadj consists of two register files with an organization of 8 -bit $\times 7$ rows for each register file. To enable automated voltage adaptation control, the register files are hardcoded with voltage increase and voltage decrease data. The voltage increase and voltage decrease data are used for 
the supply voltage adaptation in the stimulator ASIC. Similar to data reading from the RAM_Stim, the reading process of the 8-bit data from the RAM_Vadj uses the clk_2MHz, which is the clock frequency for the Manchester data transmission.

The data transmitter control unit controls the frequency of reading the stored stimulation data from the RAM Stim according to the selected stimulation rate and the transmission of Manchester encoded data at $\sim 1 \mathrm{Mbps}$ to the stimulator ASIC using the "clk_2MHz". The backchannel decoder decodes the received pulse code signal ("gsu_lm_out") from the stimulator ASIC and activates certain flags for data reception acknowledgment and monitoring purposes.

\subsection{Data Transceiver Protocol}

Fig. 7 illustrates the data receiving and transmitting protocol of the data transceiver module from Fig. 6. There are two finite state machines (FSMs) that control the receiving and transmitting of Manchester data to and from the data transceiver module. The receiver FSM which resides in data receiver control unitconverts the serial data and controls the storage of 8-bit "regData" from the PC into the RAM Stim module. The transmitter FSM which resides in the data transmitter control unit controls the transmission of Manchester data from the data transceiver module to the stimulator ASIC. To emulate the Manchester data rate of $\sim 1 \mathrm{Mbps}$ to the stimulator ASIC, the data reception process is controlled at a higher clock speed of $100 \mathrm{MHz}$ whereas the data transmission process is controlled at a lower clock speed of $2 \mathrm{MHz}$. Therefore, the receiver FSM operates at $100 \mathrm{MHz}$ while the Transmitter FSM operates at $2 \mathrm{MHz}$.
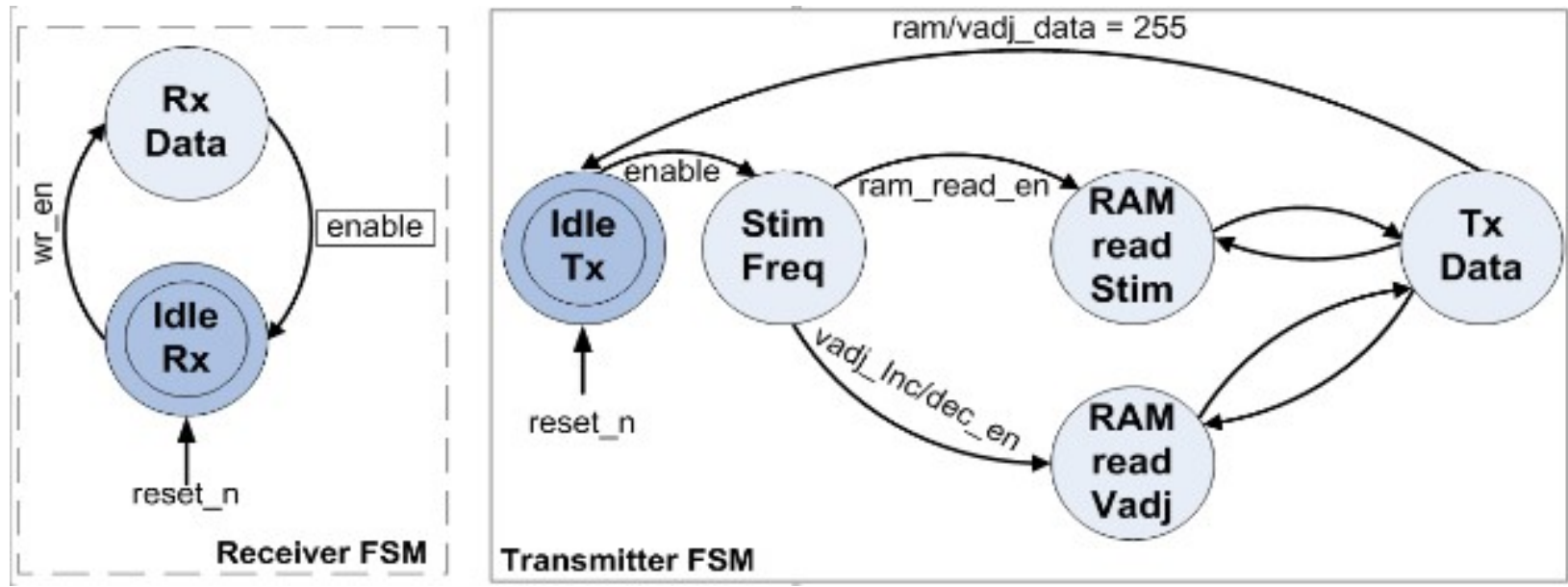

Fig.7. Data transceiver protocol

To simplify the explanation, the data transceiver protocol is explained according to its operation. 


\subsubsection{Receiver FSM}

After the system is power on reset, the receiver FSM goes to an Idle Rx state. When the stimulation data are sent from the PC to the FPGA board, the "wr en" signal is activated and the receiver FSM moves to the $\mathrm{Rx}$ data state where the received stimulation data are immediately stored into their dedicated location at the RAM Stim module. After the data reception process is completed, the "enable" flag is activated and the receiver FSM moves to the Idle Rx state.

\subsubsection{Transmitter FSM}

After the system is power on reset, the transmitter FSM goes to an Idle Tx state. When the "enable" signal is activated, the transmitter FSM moves to the Stim Freq state. In the Stim Freq state, it waits for the relevant flags ("ram read en", "vadj_inc_en", "vadj_dec_en") to be activated. For the stimulation waveform protocol, once the 16-bit internal counter has reached the required stimulation period (frequency), the "ram read en" flag is activated and the transmitter FSM moves to the RAM read Stim state. In this RAM read Stim state, the 8-bit "ram_data" from RAM Stim module is read and loaded into the internal 8-bit register of the Data Transmitter Control Unit. Then, the transmitter FSM moves to the Tx data state where the parallel 8-bit is converted into serial data before it is transmitted serially to the stimulator ASIC. The process of reading the 8-bit "ram_data" and transmitting the data serially is repeated until the "ram_data" indicates the last stored value of 255. The transmitter FSM will then move from the Tx Data to the Idle Tx state.

For the supply voltage adaptation protocol, when the stimulator ASIC is powered up, the supply voltage $\left(\mathrm{V}_{\mathrm{DDP}}\right)$ is set to its maximum level. During the stimulation process, if no voltage compliance problem is reported from the stimulator ASIC after $100 \mathrm{~ms}$, the data transmitter control unit module activates the "vadj_dec_en" flag. Once the "vadj_dec_en" flag is activated, the FSM moves from the Stim Freq state to the RAM read Vadj state. At the RAM read Vadj state, the 8-bit voltage decrease data is read from the RAM Vadj module and is loaded into the internal 8-bit register of the data transmitter control unit. The transmitter FSM then moves to the Tx data state for the serial data transmission. Similarly, the process of reading and transmitting the 8-bit voltage decrease data serially is repeated until it indicates the last stored data have the value of 255. The transmitter FSM will then move from the Tx Data to the idle Tx and Stim Freq states and waits for the next stimulation cycle to begin. Therefore, the supply voltage is decreased by transmitting the voltage decrease data to the stimulator ASIC. The voltage decrease data is repeatedly transmitted to the stimulator ASIC every $100 \mathrm{~ms}$ if no voltage compliance problem is 
detected. Thus, the transmitter FSM will repeat executing the RAM read Vadj and Tx Data states. However, if a voltage compliance problem is detected in the stimulator ASIC, the "compl_err_reg" flag is activated $1.6 \mathrm{~ms}$ after the reception of the pulse code signal ("gsu_lm_out") from the stimulator ASIC. The "vadj_inc_en" flag is then activated by the data transmitter control unit module. Therefore, the transmitter FSM moves from the Stim Freq state to the RAM read Vadj state when the "vadj_inc_en" flag is activated. In the RAM read Vadj state, the 8-bit voltage increase data is read from the RAM Vadj module and loaded into the internal 8-bit register of the data transmitter control unit. Similarly, the transmitter FSM then moves to the Tx data state for the serial data transmission. The process of reading and transmitting the 8-bit voltage increase data serially is repeated until it indicates the last stored data have the value of 255 . The transmitter FSM will then move from the Tx data state to the idle Tx and Stim Freq states and wait for the next stimulation cycle to begin. The supply voltage of the stimulator ASIC is increased accordingly after the reception of voltage increase data and is assumed to have reached an optimum voltage compliance after the first compliance problem is detected. Thereafter, the supply voltage decrement process is only monitored every 1s. Therefore, the transmitter FSM will only enter the RAM read Vadj state after 1s. However, if a subsequent voltage compliance problem is detected, the transmitter FSM will then repeatedly execute the RAM read Vadj and Tx data states for the voltage increase data reading and transmission. Thus, the voltage increase data is transmitted again to the stimulator ASIC for the voltage increment process.

\section{RESULTS AND DISCUSSION}

This section discusses a few of the simulation results from the ECU's data transceiver unit and the real-time measurement results of the stimulator ASIC testing using the ECU.

\subsection{ECU's Data Transceiver Simulation Results}

Two important simulation results of the ECU's data transceiver are discussed here. The first simulation results illustrate and discuss on the data transceiver operation for serial Manchester data reception and transmitting process. The second simulation results illustrate and discuss on the backchannel decoder operation for voltage compliance problem detection and voltage adaptation process.

\subsection{Data Transceiver}

Fig. 8(a) depicts the receiving and transmitting simulation results of the ECU's data transceiver 
module. Initially, the serial data is received from the PC and temporarily stored in the RAM Stim module. Thereafter, once the "enable" signal is activated, the Manchester data is serially transmitted to the stimulator ASIC. Fig. 8(b) depicts the zoom view into marked area A of the received serial data from the $\mathrm{PC}$ and the bit shifting process into the 8-bit "regData" register. Once the received serial data is shifted and converted into 8-bit parallel data ("regData"), the 8-bit data is temporarily stored into the RAM Stim module ("ram_data") by activating the "wr_en" signal.

The zoom view into marked area B as depicted in Fig. 8(c), indicates the serial transmission of Manchester data from the transmitter control unit. It is observed that the parallel 8-bit "ram_data" from the RAM Stim register file is converted into serial Manchester data at $\sim 1 \mathrm{Mbps}$ using $2 \mathrm{MHz}$ clock frequency. In the real test measurement setup, these serial $\sim 1 \mathrm{Mbps}$ Manchester data will be transmitted to the stimulator ASIC for stimulation programming and waveform generation.

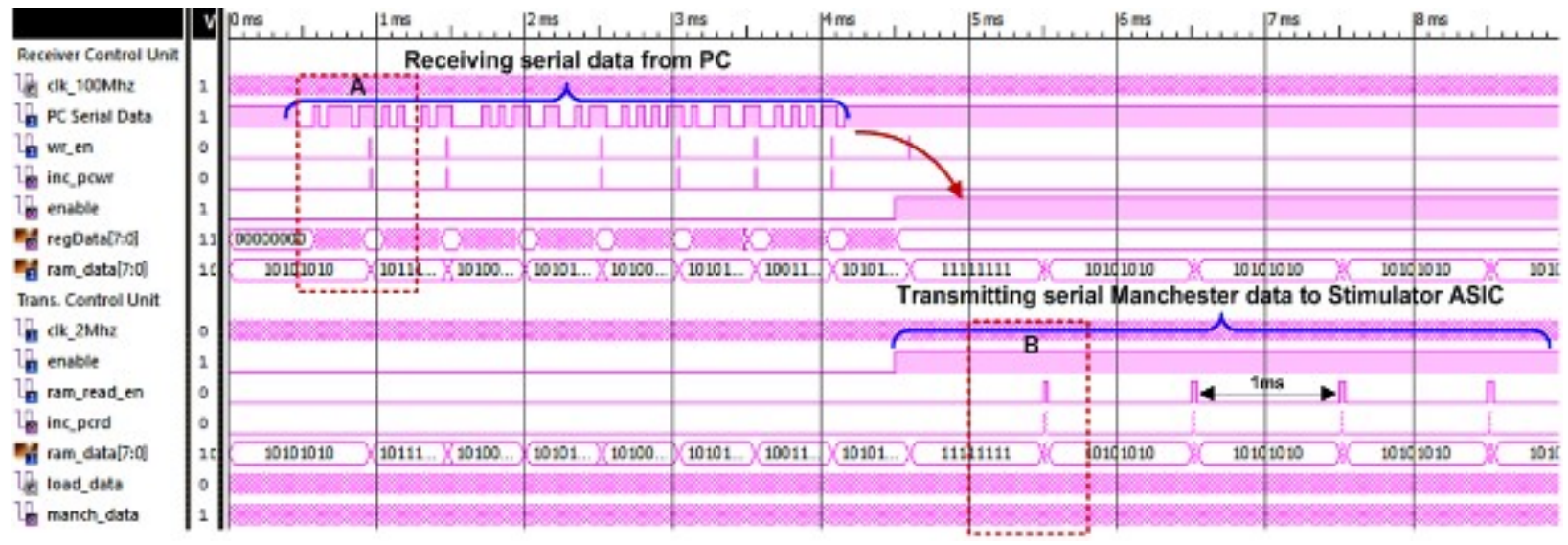

(a) Data receiving and transmitting in the data transceiver module

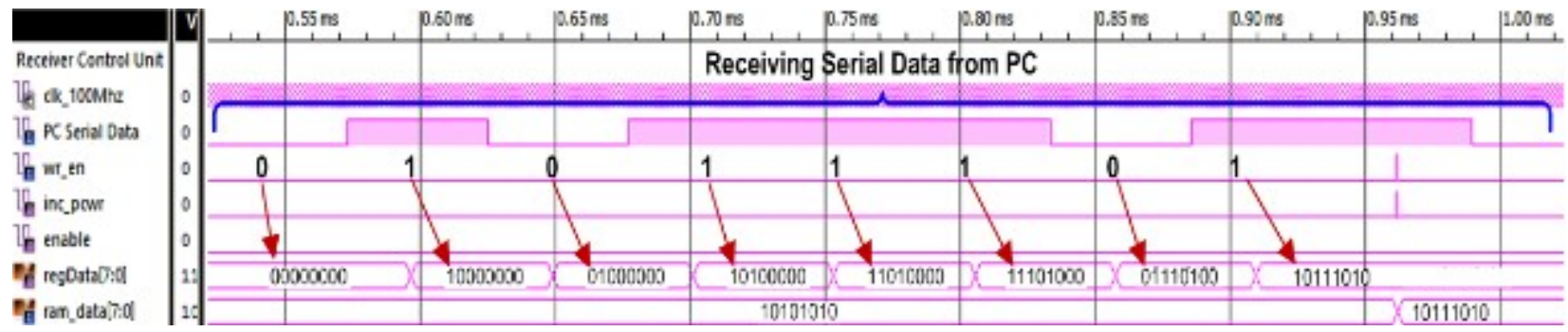

(b) Zoom view into marked area A of the receiver control unit for serial data reception from PC 


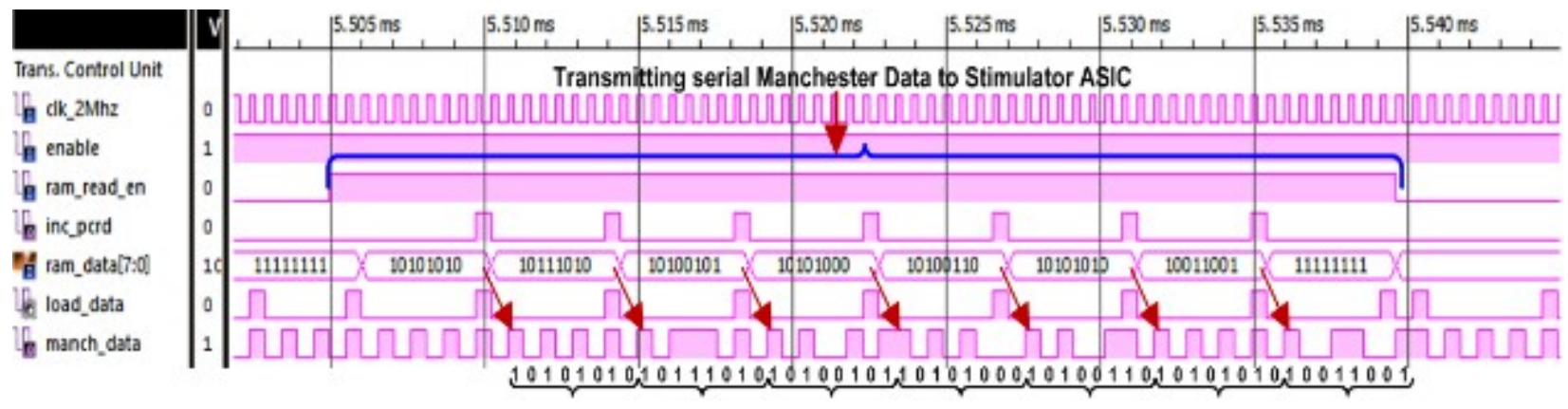

(c) Zoom view into marked area B of the transmitter control unit for serial Manchester data

$$
\text { transmission at } \sim 1 \mathrm{Mbps}
$$

Fig.8. Simulation results of data receiving and transmitting in the data transceiver module

\subsection{Backchannel Decoder}

Fig. 9(a) depicts the simulation result of the backchannel decoder module in the data transceiver. In this simulation, the voltage compliance signal is intentionally activated from the GSU (stimulator ASIC) to observe and check the functionality of the backchannel decoder module in the data transceiver. Once the voltage compliance signal ("gbl_compl") is detected by the GSU, a serial pulse code signal ("gsu_lm_out") is transmitted to the ECU from the stimulator ASIC. This serial pulse code signal ("gsu_lm_out") is decoded by the backchannel decoder. Thereafter, a “compl_err_reg" flag is activated after $1.6 \mathrm{~ms}$ to acknowledge the reception and to indicate that voltage compliance problem occurs in the stimulator ASIC.

Once the "compl_err_reg" flag is activated, the transmitter control unit will activate "vadj_inc_en" signal to read voltage increase data ("ram_vadj") from RAM Vadj module and to transmit serially to the stimulator ASIC as indicated in Fig. 9(b), the zoom view into marked area B. The voltage increase data will be used by the stimulator ASIC to increase the supply voltage ( $\left.\mathrm{V}_{\mathrm{DDP}}\right)$ accordingly. The real-time measurement results on voltage adaptation control mechanism by the ECU to the stimulator ASIC will be further illustrated and discussed in the next following section.

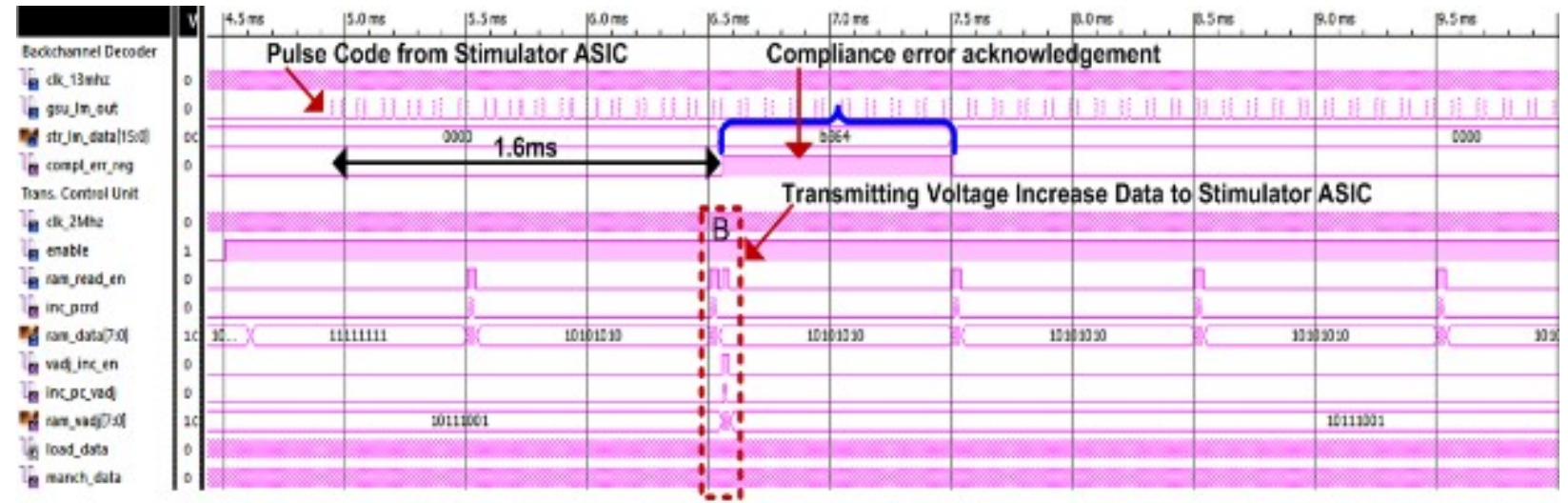


(a) Backchannel decoder in the data transceiver

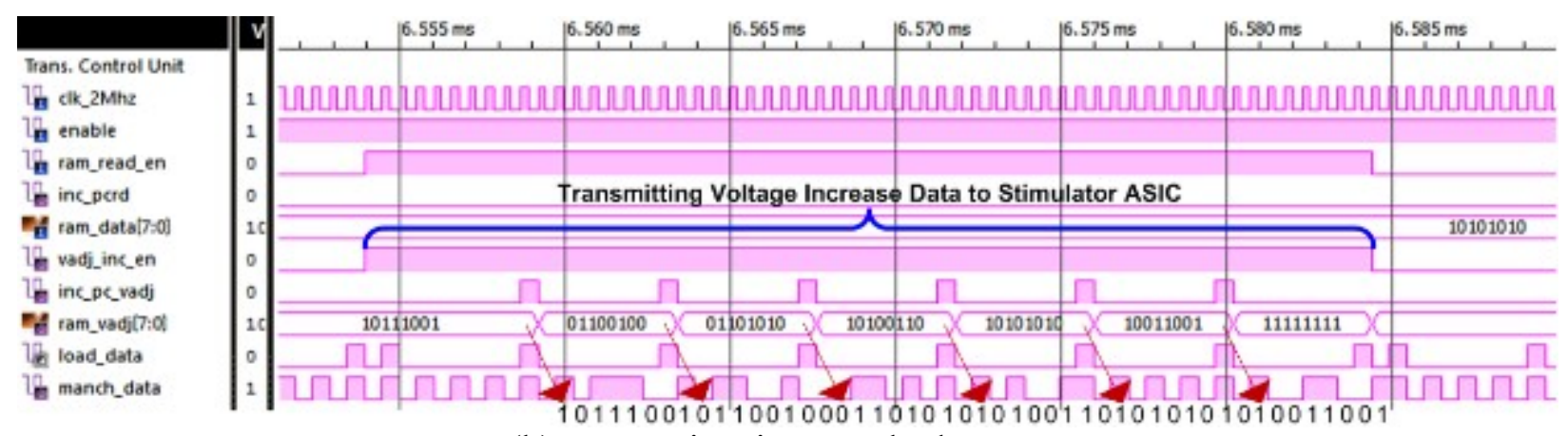

(b) Zoom view into marked area B

Fig.9. Simulation waveform of backchannel decoder

\subsection{ECU and Stimulator ASIC Measurement Results}

In this section, three samples of measurement results are shown here to illustrate the real-time stimulation operation and control mechanism of the implemented ECU with the fabricated stimulator ASIC. The first result discusses on the generation of stimulation pulse by sending the intended stimulation data to the stimulator ASIC from the ECU. The second result discusses on the decoding of the backchannel data (pulse code signal) by the ECU for voltage compliance monitoring purpose. The third result discusses the voltage adaptation control mechanism by the ECU to the stimulator ASIC for power management monitoring purposes.

\subsection{Stimulation Waveform Generation}

Fig. 10 illustrates an overview of rectangular symmetric pulse stimulation at a stimulation frequency of $60 \mathrm{~Hz}$. The internal "ram read en" signal from the data transceiver of the ECU is activated at a frequency of $60 \mathrm{~Hz}$. Therefore, the stimulation data are repeatedly read from the RAM Stim module and transmitted to the stimulator ASIC at every $16.67 \mathrm{~ms}$. As a result, the stimulation pulse is generated at the stimulation output driver every $16.67 \mathrm{~ms}$. As can be seen from Fig. 10(a), two LSUs are activated at a stimulation rate of $60 \mathrm{~Hz}$. The zoom view into the marked area A, as shown in Fig. 10(b), indicates each stimulation cycle with the relevant 5-bit "stim cmd" as listed in the Register File's timing table of the stimulator ASIC. A further zoom view into marked area B, as shown in Fig. 10(c), we can observe the "ram read en" flag is activated high and the transmission of $\sim 1 \mathrm{Mbps}$ Manchester data from the ECU before the stimulation waveform is generated at the output of stimulator ASIC. 


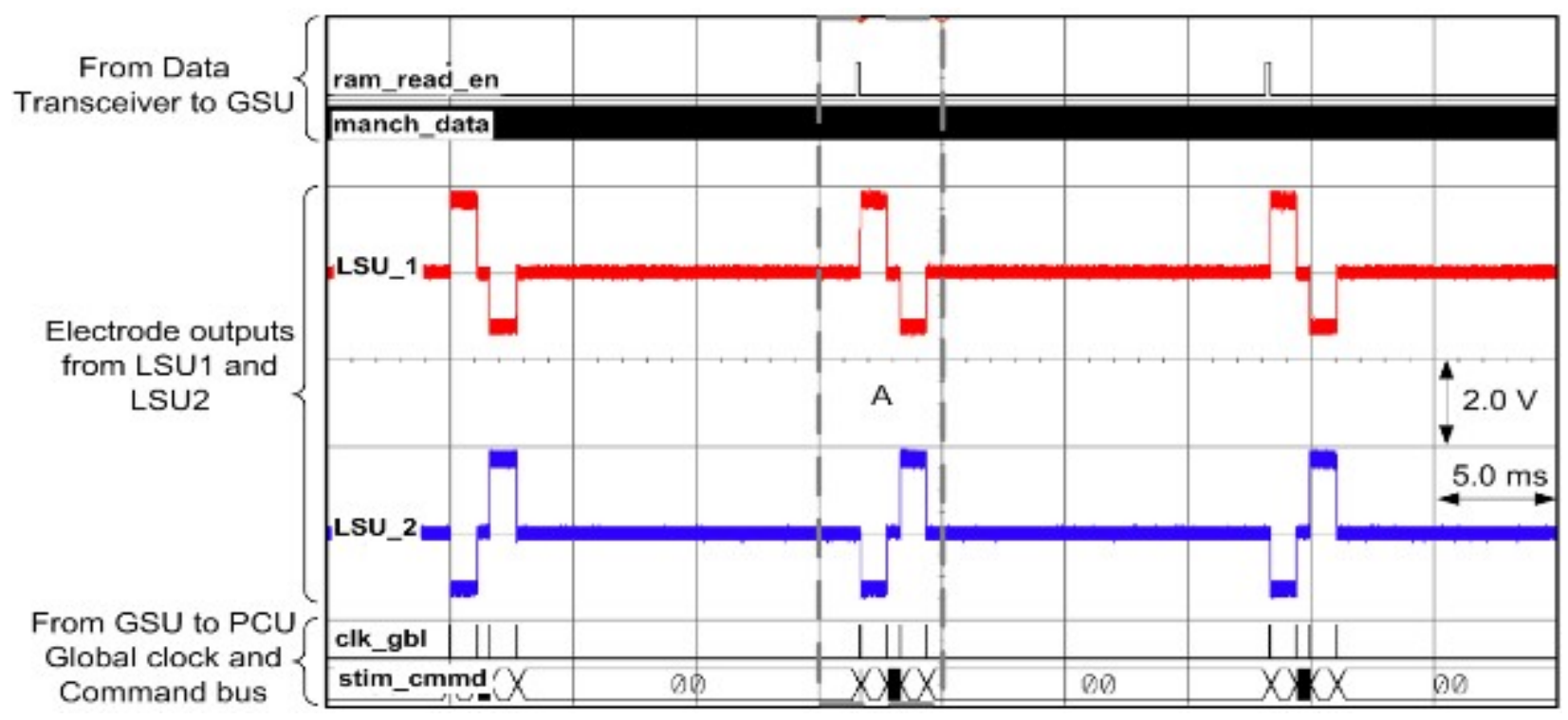

(a) Stimulation pulse of $2 \mathrm{LSU}$ at $60 \mathrm{~Hz}$ repetition

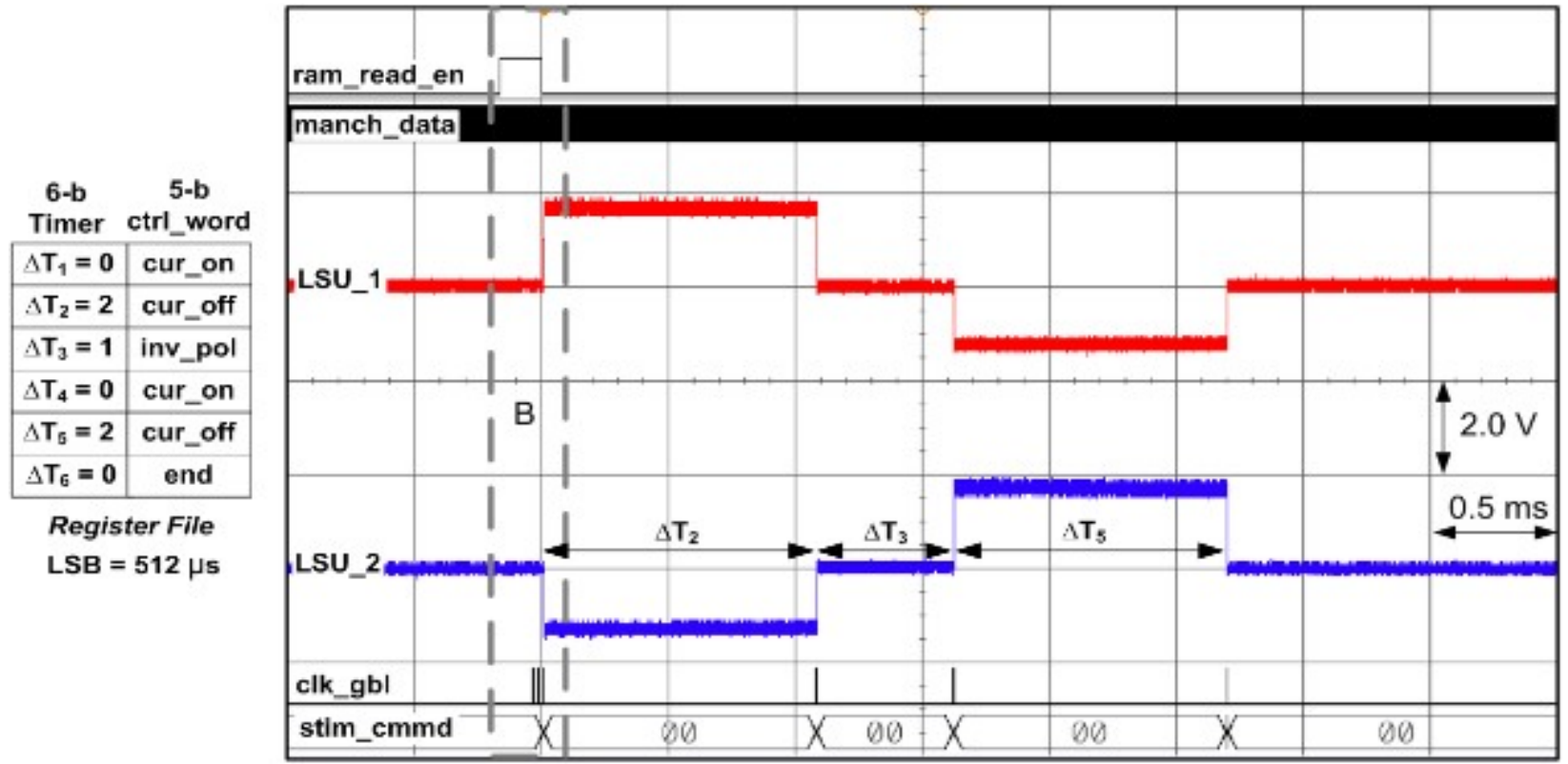

(b) Zoom view into marked area $\mathrm{A}$ for each stimulation pulse 


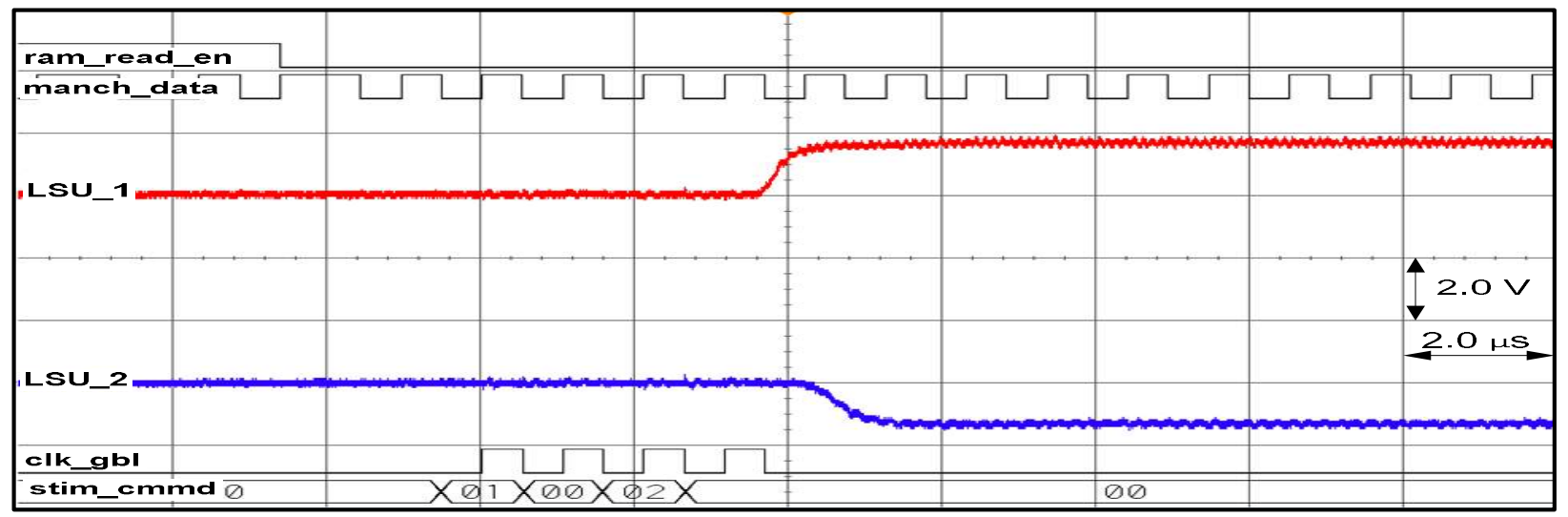

(c) Zoom view into marked area B

Fig.10. Stimulation waveform at $60 \mathrm{~Hz}$ using $\sim 1 \mathrm{Mbps}$ Manchester data from the ECU

\subsection{Voltage Compliance}

The Global Compl is used to inform the GSU of any voltage compliance problems that occur from any of the LSUs. Each LSU is equipped with a voltage compliance monitoring circuit to check the voltage compliance problem if the stimulator output voltage has reached the supply rail voltage [11]. Fig. 11 illustrates the stimulation operation with or without a voltage compliance problem. Fig. 11(a) depicts the stimulation output voltage without any voltage compliance problem and Fig. 11(b) indicates the stimulation output voltage with a voltage compliance problem. As can be seen in Fig. 11(a), because the stimulation output voltages for both LSUs have not reached the supply rail of $\pm 10 \mathrm{~V}$, the "glb compl" signal is not activated. Therefore, the backchannel module in the GSU is not activated.

However, in Fig. 11(b), the stimulator output voltage in LSU 1 has reached the supply rail, and the "glb compl" signal is activated. As a result, the GSU module is activated to send the voltage compliance problem. Then, the pulse code "gsu lm out" signal from the GSU is sent to the ECU. At the ECU's data transceiver, the received pulse code "gsu lm out" signal is decoded and the "compl err reg" flag is activated to indicate the reception of the voltage compliance problem after $1.6 \mathrm{~ms}$. From the voltage compliance monitoring feedback, the ECU will take necessary action by increasing the supply voltage of the power transmitter unit. 


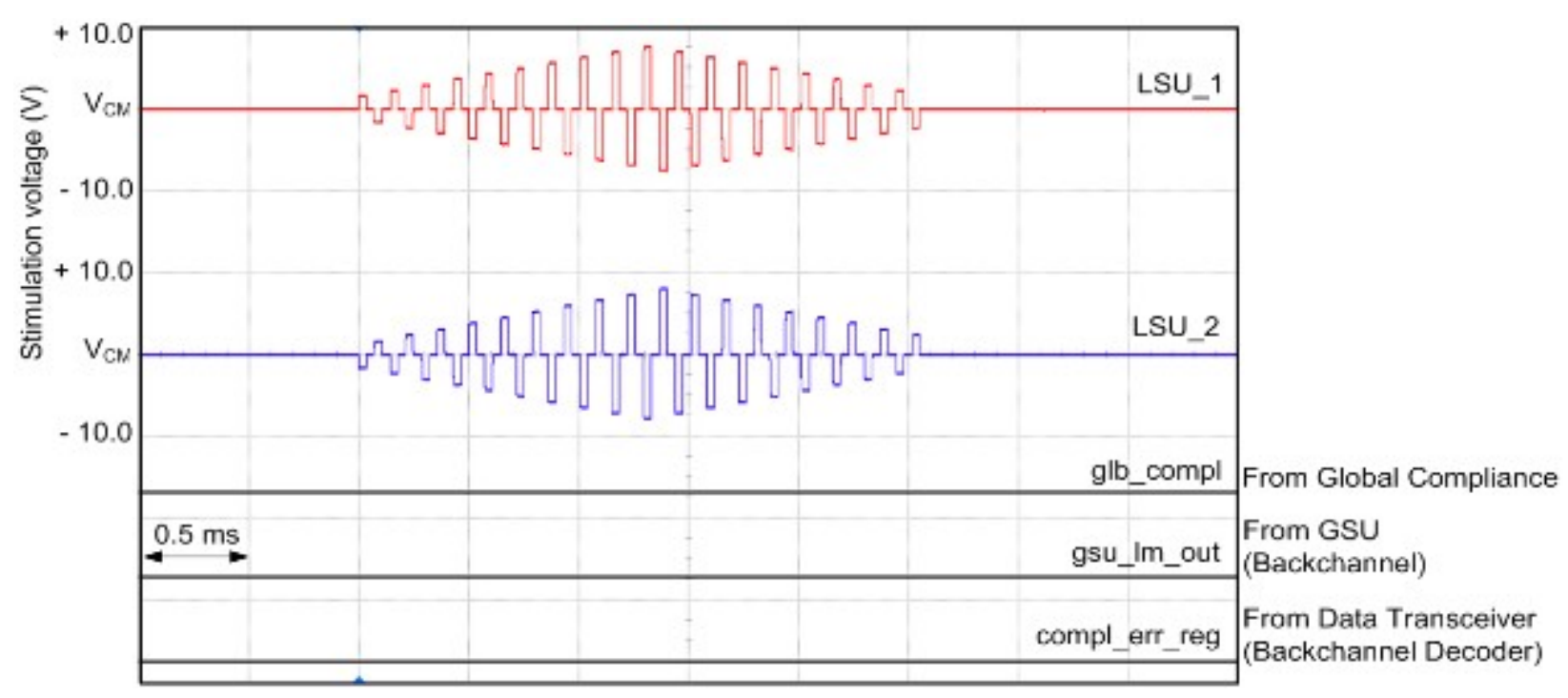

(a) Stimulation output voltage without compliance error

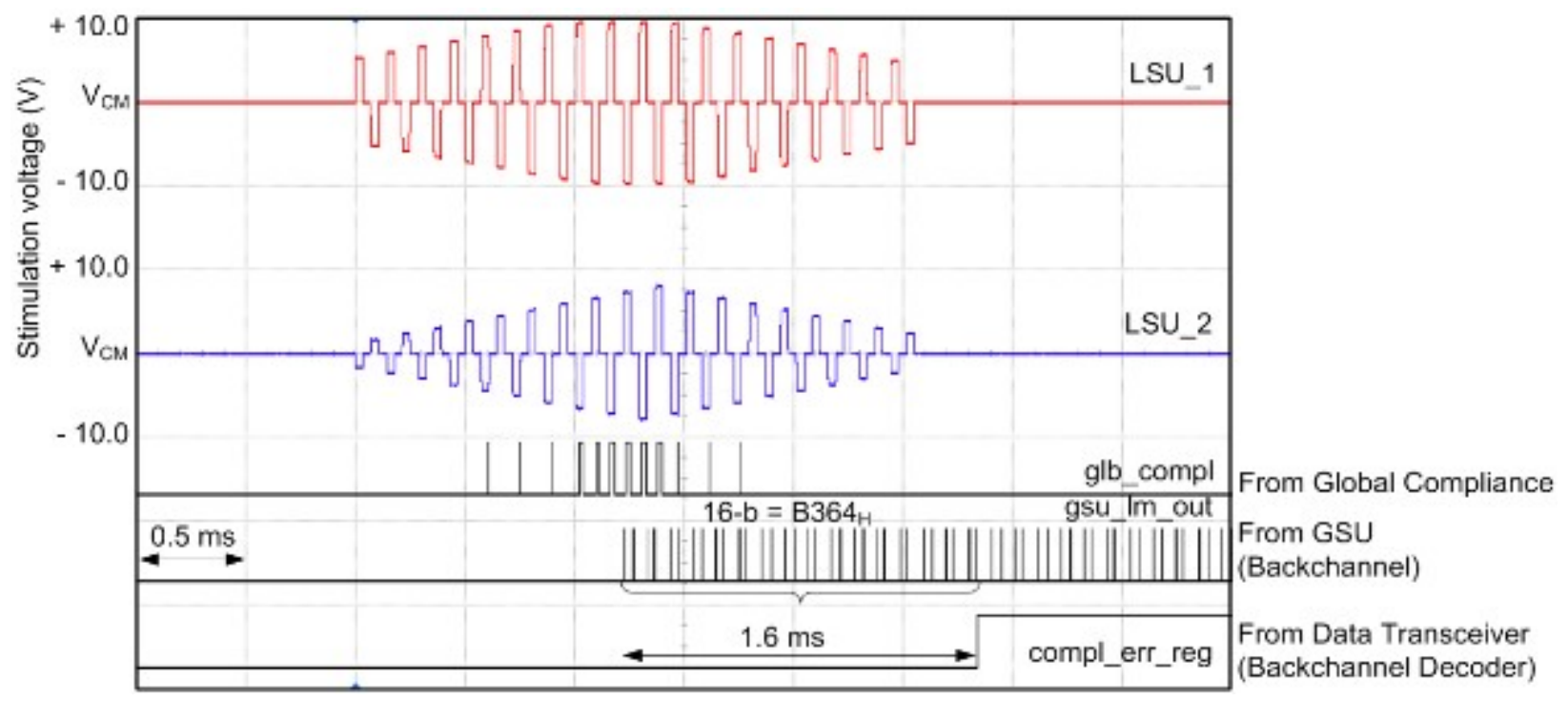

(b) Stimulation output voltage with compliance error

Fig.11. Stimulation output voltage with global compliance

\subsection{Voltage Adaptation Control}

Fig. 12 illustrates the effect of the voltage adaptation control mechanism from the ECU. The "vadj switch" register is connected to the diode switches in the shunt regulator circuit (as depicted in Fig. 2). At power on reset, the "vadj switch" register is set to binary "0000" $(0 \mathrm{H})$. Therefore, the $\mathrm{V}_{\mathrm{DDP}}$ is set at its maximum level. Thereafter, the ECU starts to monitor the compliance voltage based on the status of the decoded "compl err reg" flag in the data transceiver unit. If there is no compliance problem detected at every 100ms (note that this value was exemplary 
chosen and can be selected on the ECU), the ECU sends the voltage decrease data as indicated by the short pulse flag "vadj dec en" in Fig. 12(a) and the zoom in view into marked area A and B in Fig. 12(b) and Fig. 12(c) respectively. It should be noted that the "vadj dec en" flag indicates the duration of the voltage decrease data sent from the ECU to the stimulator ASIC.

The high supply voltage is decreased by shifting binary " 1 " into the 4-bit "vadj switch", starting from the LSB towards the MSB to turn off the diode string in the shunt regulation reference voltage (see Fig. 2). Therefore, the "vadj switch" data starts from "0000" $(0 \mathrm{H})$, "0001" $(1 \mathrm{H})$, "0011" (3H), "0111" (7H) and "1111" (FH) as indicated in Fig. 12(a) and Fig. 12(b). The binary " 1 " is shifted left into the "vadj switch" register to turn off the diode string in the power module for the voltage decrement process. The $\mathrm{V}_{\mathrm{DDP}}$ is decreased by $1.3 \mathrm{~V}$ at each decrement step when there is no compliance problem detected at every $100 \mathrm{~ms}$. Therefore, if the $\mathrm{V}_{\text {DDP }}$ starts from $13 \mathrm{~V}$, it steps down to $11.7 \mathrm{~V}, 10.4 \mathrm{~V}, 9.1 \mathrm{~V}$ and $7.8 \mathrm{~V}$ at each decrement step. Fig. 12(c) depicts the zoom in view into marked area $\mathrm{B}$ of the voltage decrement at $100 \mathrm{~ms}$ and voltage increment once the "compl err reg" is detected at the ECU. Once the voltage compliance problem is detected, the $\mathrm{V}_{\mathrm{DDP}}$ is increased by sending voltage increase data from the ECU to the stimulator ASIC as indicated by the "vadj inc en" flag. As can be seen from Fig. 12(c), once the compliance error problem is decoded and detected by the ECU's Data Transceiver, the "compl err reg" flag is activated until the next stimulation cycle begins. However, the "vadj inc en" flag is activated for a short duration after the rising edge of the "compl err reg" flag. This process is to ensure that the voltage increase data are sent only once after the detection of the "compl err reg" flag from the ECU. The binary " 0 " is shifted right into the "vadj switch" register, which results in the change of binary values from "1111" (FH) to "0111" (7H) to open one diode switch. Consequently, the V VDP is increased by $1.3 \mathrm{~V}$. 


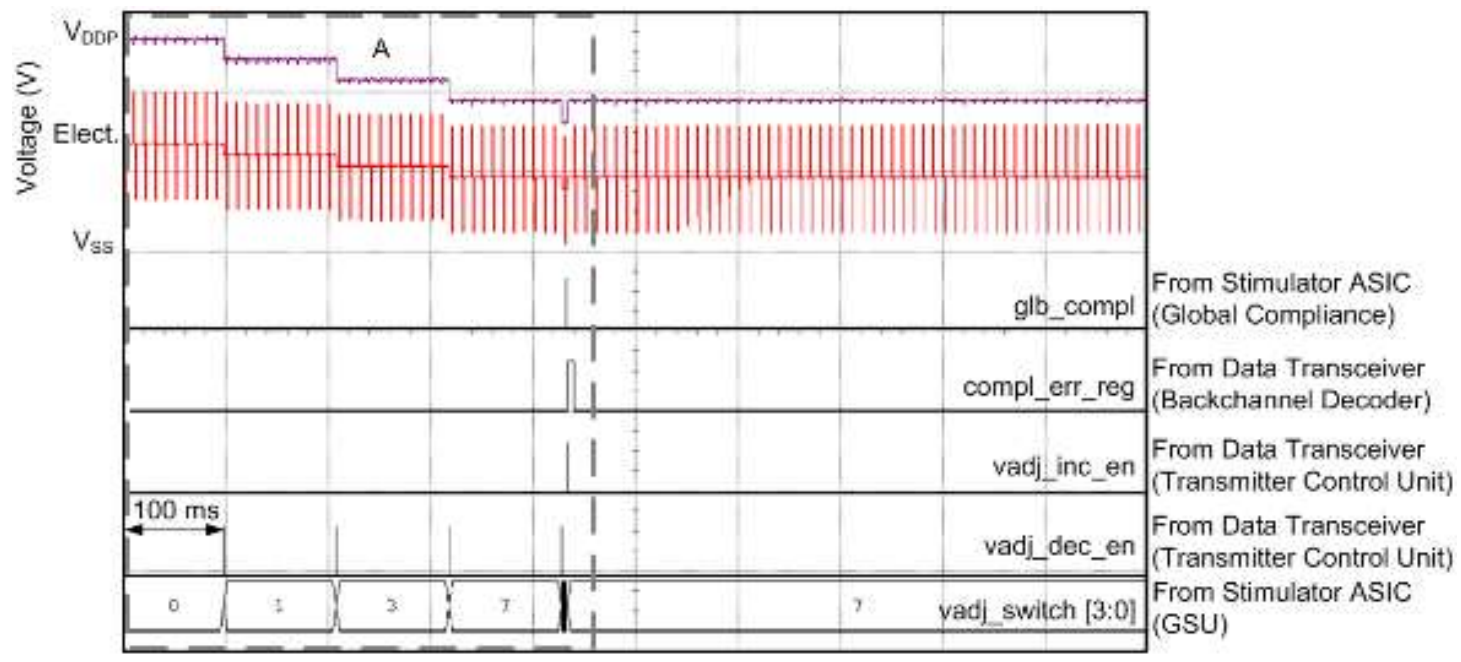

(a) Voltage adaptation control

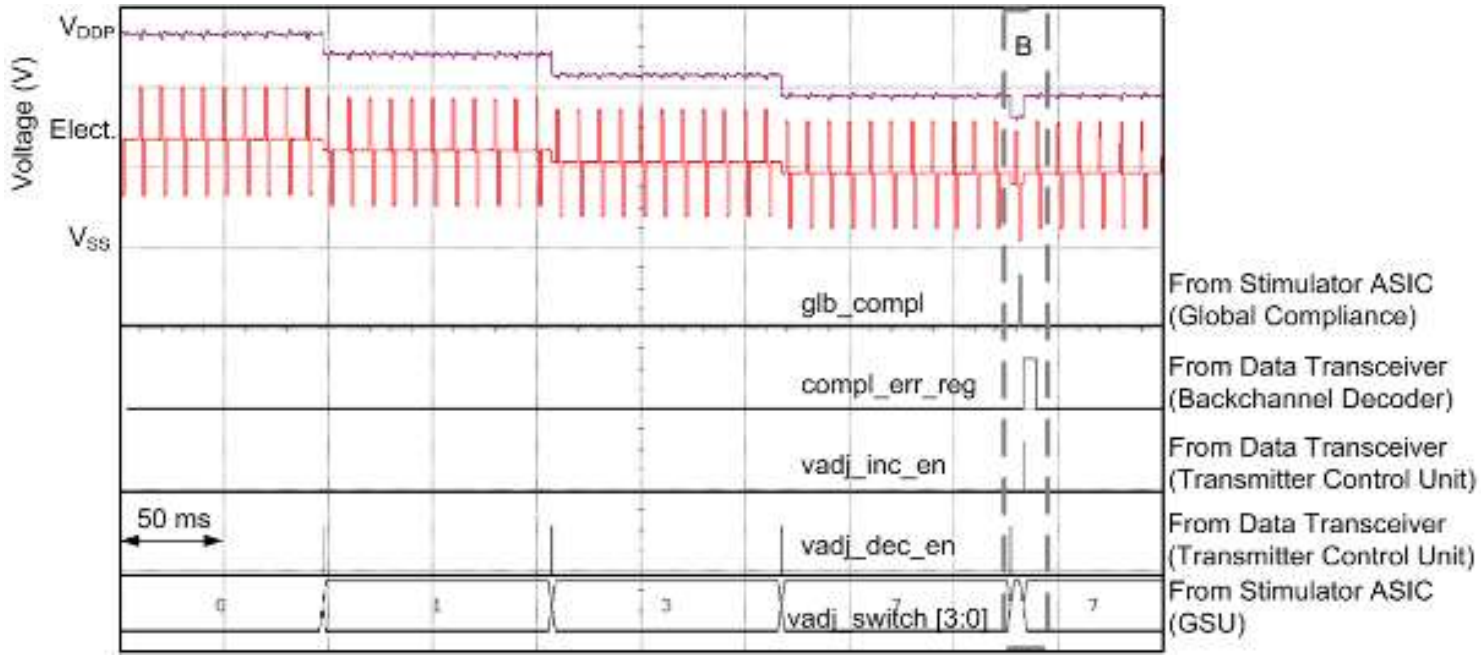

(b) Zoom view into marked area $\mathrm{A}$

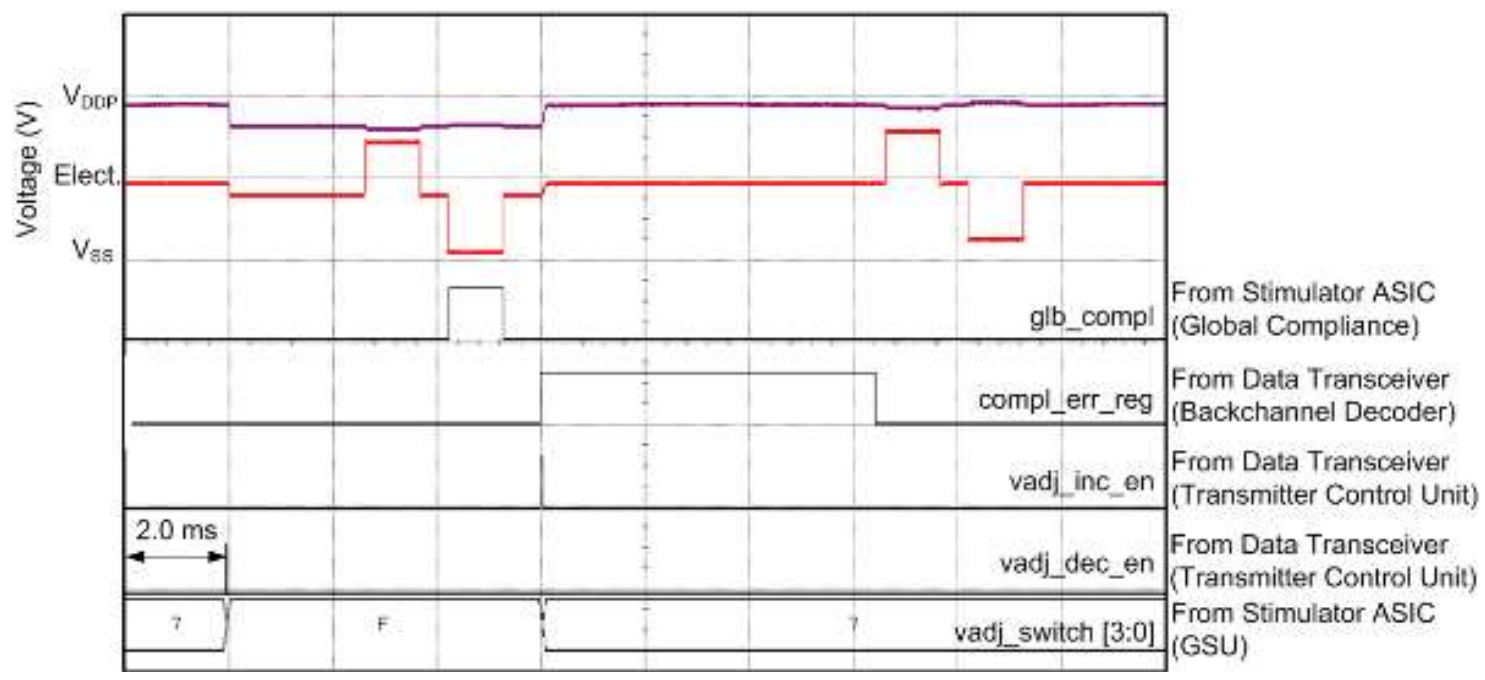

(c) Zoom view into marked area B

Fig.12. Voltage adaptation control after power start-up 


\section{CONCLUSION}

The design and development of the ECU has been illustrated and elucidated. The ECU was designed to test the functionality of the stimulator ASIC, to monitor the status of the stimulator ASIC and to provide a closed-loop external control mechanism for the stimulator ASIC supply voltage adaptation. The ECU mainly consists of the GUI from MATLAB tool for setting up the intended stimulation pattern and the data transceiver. The data transceiver was designed in HDL Verilog [21] code and was implemented in Virtex-II Pro FPGA board. The functions of the Data Transceiver are to receive serial Manchester data from the PC at a high data rate, to retransmit the Manchester data to the stimulator ASIC at a data rate of $\sim 1 \mathrm{Mbps}$, to decode serial pulse code signal or backchannel data from the stimulator ASIC for monitoring purposes and to externally control the stimulator ASIC supply voltage adaptation for power efficiency. The shown simulation and measurement results for the serial data reception from the $\mathrm{PC}$, the data transmission to the stimulator ASIC, the stimulation waveform generation, the voltage compliance monitoring and the control mechanism of voltage adaptation process validate the design functionality of the designed ECU and the stimulator ASIC.

\section{ACKNOWLEDGEMENTS}

The authors would like to thank Prof. Maurits Ortmanns from the Institute of Microelectronics, University of Ulm, Germany for the stimulator ASIC fabrication and for the test measurement setup conducted at the laboratory.The authors would also like to acknowledge the UiTM grant: 600-IRMI/DANA/5/3/ARAS (0125/2016).

\section{REFERENCES}

[1]Ortmanns M, Rocke A, Gehrke M, Tiedtke H J. A 232-channel epiretinal stimulator ASIC. IEEE Journal of Solid-State Circuits, 2007, 42(12):2946-2959

[2]Wong YT, Dommel N, Preston P, Hallum LE, Lehmann T, Lovell NH, Suaning GJ. Retinal neurostimulator for a multifocal vision prosthesis. IEEE Transactions on Neural Systems and Rehabilitation Engineering, 2007, 15(3):425-434. 
[3] Chen K, Yang Z, Hoang L, Weiland J, Humayun M, Liu W. An integrated 256-channel epiretinal prosthesis. IEEE Journal of Solid-State Circuits, 2010, 45(9):1946-1956

[4] Suaning G J, Lovell N H. CMOS neurostimulation ASIC with 100 channels, scaleable output, and bidirectional radio-frequency telemetry. IEEE Transactions on Biomedical Engineering, 2001, 48(2):248-260

[5] Sivaprakasam M, Liu W, Wang G, Weiland J D, Humayun M S. Architecture tradeoffs in high-density microstimulators for retinal prosthesis. IEEE Transactions on Circuits and Systems I: Regular Papers, 2005, 52(12):2629-2641

[6] Theogarajan L S. A low-power fully implantable 15-channel retinal stimulator chip. IEEE Journal of Solid-State Circuits, 2008, 43(10):2322-2337

[7] Sivaprakasam M, Liu W, Humayun M S, Weiland J D. A variable range bi-phasic current stimulus driver circuitry for an implantable retinal prosthetic device. IEEE Journal of Solid-State Circuits, 2005, 40(3):763-771

[8] Graf H G, Harendt C, Engelhardt T, Scherjon C, Warkentin K, Richter H, Burghartz J N. High dynamic range CMOS imager technologies for biomedical applications. IEEE Journal of Solid-State Circuits, 2009, 44(1):281-289

[9] Rothermel A, Liu L, Aryan N P, Fischer M, Wuenschmann J, Kibbel S, Harscher A. A CMOS chip with active pixel array and specific test features for subretinal implantation. IEEE Journal of Solid-State Circuits, 2009, 44(1):290-300

[10] Shire D B, Kelly S K, Chen J, Doyle P, Gingerich M D, Cogan S F, Drohan W A, Mendoza O, Theogarajan L, Wyatt J L, Rizzo J F. Development and implantation of a minimally invasive wireless subretinal neurostimulator. IEEE Transactions on Biomedical Engineering, 2009, 56(10):2502-2511

[11] Noorsal E, Sooksood K, Xu H, Hornig R, Becker J, Ortmanns M. A neural stimulator frontend with high-voltage compliance and programmable pulse shape for epiretinal implants. IEEE Journal of Solid-State Circuits, 2012, 47(1):244-256

[12] Noorsal E, Sooksood K, Xu H, Sukumaran D, Ortmanns M. Multistimulator backchannel communication link implemented for safety information and closed-loop power management. In IEEE International Conference on Control System, Computing and Engineering, 2014, pp. $647-651$ 
[13] Xu Q, Hu D, Duan B, He J. A fully implantable stimulator with wireless power and data transmission for experimental investigation of epidural spinal cord stimulation. IEEE Transactions on Neural Systems and Rehabilitation Engineering, 2015, 23(4):683-692 [14] Jiang D, Cirmirakis D, Schormans M, Perkins T A, Donaldson N, Demosthenous A. An integrated passive phase-shift keying modulator for biomedical implants with power telemetry over a single inductive link. IEEE transactions on biomedical circuits and systems, 2017, 11(1):64-77

[15] Sharmili N, Rao V B, Seetharamaiah P, Swapna N. A prototype 1024 electrode embedded computer based epiretinal prosthesis system. In IEEE 3rd International Conference on Signal Processing and Integrated Networks, 2016, pp. 337-341

[16] Liu W, Sivaprakasam M, Wang G, Zhou M, Granacki J, LaCoss J, Wills J. Implantable biomimetic microelectronic systems design. IEEE Engineering in Medicine and Biology Magazine, 2005, 24(5):66-74

[17] Coulombe J, Sawan M, Gervais J F. A highly flexible system for microstimulation of the visual cortex: Design and implementation. IEEE Transactions on Biomedical Circuits and Systems, 2007, 1(4):258-269

[18] Xu H, Noorsal E, Sooksood K, Becker J, Ortmanns M. A multichannel neurostimulator with transcutaneous closed-loop power control and self-adaptive supply. In IEEE 38th European Solid State Circuits Conference, 2012, pp. 309-312

[19] Noorsal E, Xu H, Sooksood K, Ortmanns M. Multichannel microstimulating SoC. In M. Sawan (Ed.), Handbook of biochips: Integrated circuits and systems for biology and medicine. New York: Springer, 2015, pp. 1-32

[20] Chun H, Yang Y, Lehmann T. Safety ensuring retinal prosthesis with precise charge balance and low power consumption. IEEE Transactions on Biomedical Circuits and Systems, 2014, 8(1):108-118

[21] Yeap K H, Nisar H, Chong K H, Rizman Z I, Yang C C, Yong Y T, Lai K C. Design of a keyless coded home lock system using Verilog hardware description language. International Journal of Electronics, Computer and Communications Technologies, 2012, 3(1):10-16 
[22] Nawi B, Sulaini B, Mohd Z A, Shamsul A Z, Zairi I R. PID voltage control for DC motor using MATLAB Simulink and Arduino microcontroller. Journal of Applied Environmental and Biological Sciences, 2015, 5(9):166-173

[23] Miskon M T, Rizman Z I, Chek W A, Fauzi F D. Fitness cycling device with graphical user interface based on IEEE 802.15. 4 transceiver for real time monitoring.Journal of Applied Environmental and Biological Sciences, 2014, 4(12):108-114

\section{How to cite this article:}

Noorsal E, Sooksood K, Xu H, Rizman Z I. An external control unit implemented for stimulator asic testing. J. Fundam. Appl. Sci., 2017, 9(6S), 710-734. 